\title{
Sonoporation-Mediated Gene Transfection: A Novel Direction for Cell Reprogramming In Vivo
}

\author{
Meng $D u^{1,2}$, Yue $L i^{1,2,3}$ and Zhiyi Chen ${ }^{1,2 *}$ \\ ${ }^{1}$ The First Affiliated Hospital, Medical Imaging Centre, Hengyang Medical School, University of South China, Hengyang, China, \\ ${ }^{2}$ Institute of Medical Imaging, Hengyang Medical School, University of South China, Hengyang, China, \\ ${ }^{3}$ Laboratory of Ultrasound Molecular Imaging, The Third Affiliated Hospital of Guangzhou Medical University, Guangzhou, China
}

Keywords: ultrasound, cell reprogramming, gene delivery, regenerative medicine, physical stimulation

\section{INTRODUCTION}

Cell reprogramming is of great significance to biomedical science, especially in regenerative medicine application. The classical mode called indirect reprogramming is to obtain stem cells or induced pluripotent stem cells (iPSCs) and stimulate them to become mature differentiated cells, which has been applied in cardiac disease, neuron disease, etc. For example, with transfection of four transcriptional factors Oct4, Sox2, Klf4, and c-Myc (Yamanaka factors), somatic cells could be reprogrammed into iPSCs and further be stimulated to differentiate into mature functional cells (Takahashi and Yamanaka, 2006; Takahashi et al., 2007).

In contrast with indirect reprogramming, direct reprogramming has received lots of attention in recent years, due to its unique advantage of fast, efficient, and low risk of tumorigenesis. Direct reprogramming refers to the application of transcription factor overexpression, noncoding RNA delivery, or small molecule delivery to promote the direct transformation of cells into target differentiated cells. In other words, compared with indirect reprogramming, direct reprogramming does not have to go through the stage of pluripotent stem cells or progenitor cells, which means it is easier to perform during cell differentiation. Lately, direct reprogramming has been applied as a therapeutic strategy to many diseases. For example, in ophthalmology, with application of five small molecules, Mahato et al. successfully reprogrammed fibroblasts into rod photoreceptor-like cells in vitro, which were further transplanted to restore vision of blind mice (Mahato et al., 2020). In another study, direct reprogramming was applied to wound healing in vivo. Kurita et al. (2018) proved that with the introduction of a combination of four epidermal growthrelated factors into the wound site, cells inside the wound were reprogrammed into epidermal cells, which enabled non-invasive wound healing and the function of new born skin was the same as the normal skin.

The greatest value of direct reprogramming is that it makes cell reprograming in vivo possible to perform (Sekiryu and Matsuda, 2021). The common method for in vivo reprograming rely on virusbased strategy, which transfect transcriptional factor gene into targeted cells. The most common used vector for gene transfection could be classified as viral and nonviral vectors (Chong et al., 2021). Viral vector has the advantage of high efficiency of gene transfection, while exogenous genes may be inserted randomly into the host cell genome, which threatens the integrity and safety of the cell genome (Geis et al., 2017). Nonviral vectors, such as liposome and nanoparticle, could reduce the rate of host immunogenicity response, which has received more concerns recently. Wang et al. developed a novel nonviral delivery system for cardiac reprograming, which consists of nanoparticles and biofilm. In detail, to enhance the targeting efficiency of this system, mesoporous silicon nanoparticles were decorated with neutrophil-mimicking membranes, which was modified with folic acid peptide. With high efficiency delivery of miRNA1, 133, 208, and 499 in vivo, cardiac fibroblasts in myocardial 
infarction area could be reprogramed into induced cardiomyocyte-like cells, and cardiac function of injury area finally recovers (Wang et al., 2021). However, compared with viral vectors, relatively low transfection efficiency limited the application of the nonviral vector.

Sonoporation is a special type of nonviral physical transfection strategy. Compared with other physical transfection modes, such as electroporation and gene microinjection, sonoporation has shown great application potential for gene transfection in vivo, due to the advantage of high specificity, deep penetration, and low cost (Tachibana et al., 1999). With gas-filled microbubbles (MBs) as cavitation nuclei and gene vector, ultrasound, able to trigger MBs rapidly oscillating and disrupt cell membrane to form transient pores (sonoporation), then enhances cellular membrane permeability and promotes gene entering into cytoplasm (Helfield et al., 2016; Rong et al., 2018; Meng et al., 2019). Sonoporation was considered as a non-invasive and nonviral approach to deliver functional molecules, such as pDNA, siRNA, or molecular drug, to overcome the physiological or pathological barriers for the purpose of preventing or treating diseases (Delalande et al., 2015; Bez et al., 2018; Tran et al., 2019).

With sonoporation, gene in acoustic responsive vector, such as $\mathrm{MBs}$, could be release quickly and enter into targeted tissue or cells. Based on this theory, sonoporation-based direct reprogramming strategy was applied in diabetes treatment. In this research, Yang et al. applied SonoVue microbubble, which is a kind of commercial contrast agent used in clinics, as vector for hydrodynamic gene Pdx1, Neurog3, and MafA. First of all, in order to optimize the transfection rate, related parameters, including acoustic pressure, pulse repetition frequency, and plasmid concentration were tested and the acoustic parameter was set at $1 \mathrm{MPa}$ acoustic pressure, 20 cycle pulses, and the irradiation duration was set at $5 \mathrm{~min}$ with an interval of $6 \mathrm{~s}$. With the ultrasound irradiation at the liver after the gene-loaded MBs administration, it was shown that the hepatocytes have been reprogrammed into insulin-producing cells in a diabetic model successfully. After treatment, the glucose levels decreased significantly while the insulin levels were enhanced, which indicated that diabetic symptoms were alleviated (Yang et al., 2020). Accordingly, sonoporation was successfully applied in direct cell reprograming in vivo, and provide a non-invasive, safe, and efficient reprograming mode for regenerative medicine.

Nowadays, with the development of bioengineering science, ultrasound could not only be applied as a diagnosis method for clinics but also get more attention in its application in biological function regulation, substance triggered release, ablation, and so on. The continuous maturation of sonoporation technology also promotes its application in clinics (Carpentier et al., 2016; Dimcevski et al., 2016; Sitta and Howard, 2021). However, as for cell reprogramming applications, sonoporation still has its limitation. First of all is the choice of gene vector. During the process of sonoporation, vector is not only used to carry genes, but also play the role as cavitation nucleus to improve permeability of tissue or cells during ultrasound irradiation. Application of commercial MBs, such as SonoVue, is a potential solution. In many clinical trials involving sonoporation-mediated drug delivery, MBs are administered separately from chemotherapy drugs. However, it is not appropriate for gene transfection since genes are easily degraded during circulation. Although a lot of novel vectors with high gene loading were developed, the biocompatibility of these chemically synthesized vectors is still a problem to be solved. Research on inducing cell reprogramming by facilitating small molecules into target tissues through sonoporation may be an interesting topic. Second is the standardization of sonoporation operation mode. Sonoporation transfection involves many key parameters, including acoustic pressure, frequency, irradiation time, and so on. The optimal parameters reported by different researches vary greatly, which may be caused by the various ultrasound devices applied. For example, the optimal parameters used in the above research (1 MPa acoustic pressure, 20 cycle pulses, $5 \mathrm{~min}$ for irradiation duration, and interval of $6 \mathrm{~s}$ ) are quite different from those used in our previous research (Yu et al., 2018; Li et al., 2021) (0.6 MPa, $30 \%$ duty cycle, and $5 \mathrm{~min}$ for irradiation duration; $2.0 \mathrm{~W} / \mathrm{cm}^{2}$, $50 \%$ duty cycle, and $1 \mathrm{~min}$ for irradiation duration). Therefore, the operation mode of sonoporation is difficult to be unified and requires individual customization. Last but not the least, the transfection efficiency. Cell reprograming, especially in vivo cell reprograming, has high requirements on gene transfection efficiency. There is still a big gap in transfection rate between virus strategy and sonoporation. Combining sonoporation with other transfection strategies, such as virus transfection and chemical transfection, is a potential solution. In addition, in order to optimize transfection efficiency, it is valuable to explore the influence of biological effects of ultrasound on mitochondria and cell fate, and reveal its biological mechanism. In other words, although sonoporation transfection still faces many challenges, based on the integration and collaboration of various disciplines (Huang, 2020), there is no doubt that sonoporation will open a new chapter for regenerative medicine in the clinic.

\section{AUTHOR CONTRIBUTIONS}

MD was responsible for the manuscript writing and literature searching. YL was responsible for the literature searching and helping in the manuscript revision. ZC designed the core idea and the theme of this manuscript, and helped in revising the manuscript.

\section{FUNDING}

This work was supported by the National Key R\&D Program of China (2019YFE0110400), National Natural Science Foundation of China (81671707, 81971621, 82102087), Natural Science Foundation of Guangdong Province (No. 2021A1515011177), Natural Science Foundation of Guangdong Province (No. 2020A1515110628), and Natural Science Foundation of Guangdong Province (No. 2019A1515012212). 


\section{REFERENCES}

Bez, M., Kremen, T. J., Tawackoli, W., Avalos, P., Sheyn, D., Shapiro, G., et al. (2018). Ultrasound-Mediated Gene Delivery Enhances Tendon Allograft Integration in Mini-Pig Ligament Reconstruction. Mol. Ther. 26 (7), 1746-1755. doi:10.1016/j.ymthe.2018.04.020

Carpentier, A., Canney, M., Vignot, A., Reina, V., Beccaria, K., Horodyckid, C., et al. (2016). Clinical Trial of Blood-Brain Barrier Disruption by Pulsed Ultrasound. Sci. Transl Med. 8 (343), 343re2. doi:10.1126/scitranslmed.aaf6086

Chong, Z. X., Yeap, S. K., and Ho, W. Y. (2021). Transfection Types, Methods and Strategies: A Technical Review. PeerJ 9, e11165. doi:10.7717/peerj.11165

Delalande, A., Leduc, C., Midoux, P., Postema, M., and Pichon, C. (2015). Efficient Gene Delivery by Sonoporation Is Associated with Microbubble Entry into Cells and the Clathrin-Dependent Endocytosis Pathway. Ultrasound Med. Biol. 41 (7), 1913-1926. doi:10.1016/j.ultrasmedbio.2015.03.010

Dimcevski, G., Kotopoulis, S., Bjånes, T., Hoem, D., Schjøtt, J., Gjertsen, B. T., et al. (2016). A Human Clinical Trial Using Ultrasound and Microbubbles to Enhance Gemcitabine Treatment of Inoperable Pancreatic Cancer. J. Control. Release 243, 172-181. doi:10.1016/j.jconrel.2016.10.007

Geis, F. K., Galla, M., Hoffmann, D., Kuehle, J., Zychlinski, D., Maetzig, T., et al. (2017). Potent and Reversible Lentiviral Vector Restriction in Murine Induced Pluripotent Stem Cells. Retrovirology 14 (1), 34. doi:10.1186/s12977-017-0358-1

Helfield, B., Chen, X., Watkins, S. C., and Villanueva, F. S. (2016). Biophysical Insight into Mechanisms of Sonoporation. Proc. Natl. Acad. Sci. USA 113 (36), 9983-9988. doi:10.1073/pnas.1606915113

Huang, P. (2020). An Integrated Approach to Ultrasound Imaging in Medicine and Biology. BIO Integration 1 (2), 105-109. doi:10.15212/bioi-2020-0036

Kurita, M., Araoka, T., Hishida, T., O’Keefe, D. D., Takahashi, Y., Sakamoto, A., et al. (2018). In Vivo reprogramming of Wound-Resident Cells Generates Skin Epithelial Tissue. Nature 561 (7722), 243-247. doi:10.1038/s41586-018-0477-4

Li, Y., Du, M., Fang, J., Zhou, J., and Chen, Z. (2021). UTMD Promoted Local Delivery of miR-34a-Mimic for Ovarian Cancer Therapy. Drug Deliv. 28 (1), 1616-1625. doi:10.1080/10717544.2021.1955041

Mahato, B., Kaya, K. D., Fan, Y., Sumien, N., Shetty, R. A., Zhang, W., et al. (2020). Pharmacologic Fibroblast Reprogramming into Photoreceptors Restores Vision. Nature 581 (7806), 83-88. doi:10.1038/s41586-020-2201-4

Meng, L., Liu, X., Wang, Y., Zhang, W., Zhou, W., Cai, F., et al. (2019). Sonoporation of Cells by a Parallel Stable Cavitation Microbubble Array. Adv. Sci. 6 (17), 1900557. doi:10.1002/advs.201900557

Rong, N., Zhou, H., Liu, R., Wang, Y., and Fan, Z. (2018). Ultrasound and Microbubble Mediated Plasmid DNA Uptake: A Fast, Global and Multi-Mechanisms Involved Process. J. Control. Release 273, 40-50. doi:10.1016/j.jconrel.2018.01.014

Sekiryu, H., and Matsuda, T. (2021). In Vivo Direct Reprogramming as a Therapeutic Strategy for Brain and Retina Repair. Neural Regen. Res. 16 (10), 1998-1999. doi:10.4103/1673-5374.308093
Sitta, J., and Howard, C. M. (2021). Applications of Ultrasound-Mediated Drug Delivery and Gene Therapy. Int. J. Mol. Sci. 22 (21), 11491. doi:10.3390/ ijms 222111491

Tachibana, K., Uchida, T., Ogawa, K., Yamashita, N., and Tamura, K. (1999). Induction of Cell-Membrane Porosity by Ultrasound. Lancet 353 (9162), 1409. doi:10.1016/s0140-6736(99)01244-1

Takahashi, K., Tanabe, K., Ohnuki, M., Narita, M., Ichisaka, T., Tomoda, K., et al. (2007). Induction of Pluripotent Stem Cells from Adult Human Fibroblasts by Defined Factors. Cell 131 (5), 861-872. doi:10.1016/ j.cell.2007.11.019

Takahashi, K., and Yamanaka, S. (2006). Induction of Pluripotent Stem Cells from Mouse Embryonic and Adult Fibroblast Cultures by Defined Factors. Cell 126 (4), 663-676. doi:10.1016/j.cell.2006.07.024

Tran, D. M., Zhang, F., Morrison, K. P., Loeb, K. R., Harrang, J., Kajimoto, M., et al. (2019). Transcutaneous Ultrasound-Mediated Nonviral Gene Delivery to the Liver in a Porcine Model. Mol. Ther. Methods Clin. Dev. 14, 275-284. doi:10.1016/j.omtm.2019.07.005

Wang, Q., Song, Y., Chen, J., Li, Q., Gao, J., Tan, H., et al. (2021). Direct In Vivo Reprogramming with Non-Viral Sequential Targeting Nanoparticles Promotes Cardiac Regeneration. Biomaterials 276, 121028. doi:10.1016/ j.biomaterials.2021.121028

Yang, X.-F., Wang, H.-Y., Lu, W.-L., Ma, W., Zhang, H., and Li, F.-R. (2020) Direct Reprogramming of Hepatocytes into Insulin-Producing Cells for Anti-Diabetic Treatment by Ultrasound-Targeted Microbubble Destruction Enhanced Hydrodynamic Gene Delivery. Am. J. Transl. Res. 12 (11), 7275-7286.

Yu, J., Chen, Z., Li, Y., Du, M., Yan, F., and Zheng, H. (2018). Echogenic Chitosan Nanodroplets for Spatiotemporally Controlled Gene Delivery. J. Biomed. Nanotechnol 14 (7), 1287-1297. doi:10.1166/jbn.2018.2575

Conflict of Interest: The authors declare that the research was conducted in the absence of any commercial or financial relationships that could be construed as a potential conflict of interest.

Publisher's Note: All claims expressed in this article are solely those of the authors and do not necessarily represent those of their affiliated organizations, or those of the publisher, the editors, and the reviewers. Any product that may be evaluated in this article, or claim that may be made by its manufacturer, is not guaranteed or endorsed by the publisher.

Copyright (C) $2022 \mathrm{Du}, \mathrm{Li}$ and Chen. This is an open-access article distributed under the terms of the Creative Commons Attribution License (CC BY). The use, distribution or reproduction in other forums is permitted, provided the original author(s) and the copyright owner(s) are credited and that the original publication in this journal is cited, in accordance with accepted academic practice. No use, distribution or reproduction is permitted which does not comply with these terms. 